\title{
LOS SISTEMAS DE BICICLETA PÚBLICA Y LA MOVILIDAD URBANA SOSTENIBLE. UN ANÁLISIS EN LA CIUDAD DE PALMA (MALLORCA, ISLAS BALEARES) ${ }^{1}$
}

\author{
Joana Maria Seguí Pons \\ Jaume Mateu Lladó \\ Maurici Ruiz Pérez \\ Maria Rosa Martínez Reynés ${ }^{2}$ \\ Departament de Geografia. Universitat de les Illes Balears \\ joana.segui-pons@uib.es
}

\section{RESUMEN}

El artículo analiza los sistemas de bicicleta pública como instrumento de incentivación de los desplazamientos en bicicleta en el marco de las políticas de movilidad sostenible. Se centra en su implantación en la ciudad de Palma y se valoran tres componentes: la evolución del reparto modal en la ciudad desde su instalación en 2011 y su relación con el tráfico ciclista; la cobertura territorial del sistema y su funcionamiento; y, por último, las características, el perfil y las motivaciones de los usuarios. Del análisis se deduce una baja aceptación del sistema, a pesar del incremento más que notable del número de ciclistas en la ciudad.

Palabras clave: movilidad sostenible, bicicleta, sistemas de bicicleta pública, Palma.

Fecha de recepción: agosto 2014.

Fecha de aceptación: octubre 2015.

1 Los datos se han obtenido en el marco del proyecto europeo CIVITAS-DYN@MO, 296057 (2012-2016). Partenariado español: Universidad de las Islas Baleares, EUROLOCAL, Ayuntamiento de Palma (Área de Movilidad), la Empresa Municipal de Transportes de Palma (EMT) y la Sociedad Municipal de Aparcamientos (SMAP). Agradecimiento especial por su apoyo en el trabajo a Felip Morell, colaborador del proyecto.

2 La profesora M. Rosa Martínez falleció el 15 de enero del 2015, descanse en paz. 


\section{ABSTRACT}

This paper aims at analyze the impact of public bike sharing systems as tools to increase bicycle trips in a Sustainable Mobility framework. To this end it develops a case study for the city of Palma, and evaluates three main issues: the evolution of the modal share in the city and its relationship with the cycling trips since the implementation of a bikesharing system in 2011; the spatial scope of the system and its main characteristics; and the profile and behavior of its users. A low acceptance follows the system, despite the significant increase in the number of cyclists in the city.

Keywords: sustainable mobility, cycling, bikesharing systems, Palma.

\section{INTRODUCCIÓN}

\section{I.1. Marco general. Los sistemas de bicicleta pública como instrumento de movilidad sosteni- ble en las ciudades}

Los sistemas de bicicleta pública han sido implementados y popularizados en muchas ciudades en los últimos años convirtiéndose en nuevos elementos de movilidad no motorizada, bien integrados en la red de transporte público (PARKES et al, 2013). En España, estos sistemas han conocido un gran auge, desde la primera implantación en la ciudad de Barcelona en el mes de marzo de 2007 (MUT, 2010). Desde entonces, son muchas las ciudades españolas que disponen de sistemas de bicicleta pública, siendo Madrid una de las últimas en incorporarla, también en la modalidad de bici eléctrica. Se perciben como una herramienta útil para el fomento de la movilidad ciclista. Tienen un efecto llamada por la comodidad y la seguridad en su uso, a pesar de los accidentes, robos o vandalismo en las bicicletas aparcadas en la calle), y por las ventajas que ofrece este modo de transporte al facilitar los viajes de carácter regular (commuters, trabajadores, o desplazamientos por otros motivos, compras, ocio, etc.) (HEINEN, VAN WEE y MAAT, 2010; GOODMAN et al, 2014).

A principios de 2014 se contabilizaron en todo el mundo alrededor de quinientos sistemas de bicicleta pública de tercera generación (MEDDIN y DE MAIO, 2014), que disponen de una red de estaciones en las que los usuarios pueden recoger las bicicletas, realizar los trayectos y depositarlas en otra estación. Para ello se disponen anclajes específicos y un sistema informático de gestión a través de tarjetas de usuarios. La literatura existente en relación a los sistemas de bicicleta pública incide generalmente en su análisis y funcionamiento. De Maio recoge la historia, evolución y características de los de tercera generación (DE MAIO, 2009); de forma similar, Parkes et al (2013) proponen un análisis de la difusión de dichas redes a nivel mundial, sobretodo, en América del Norte y Europa, a través de encuestas realizadas a sus gestores. Dell'Olio, Ibeas y Moura (2011) o Chemla, Meunier y Wolfler Calvo (2013) abundan en los aspectos más técnicos, desde el punto de vista de la ingeniería. Por otra parte, Aldred (2013) o Fishman, Washington y Haworth (2012) ofrecen un análisis de los perfiles de usuarios y de sus características principales. 
Destaca igualmente el estudio realizado por Castro (2011) sobre la contribución de estos sistemas a la movilidad sostenible en Europa. El cambio modal en favor del transporte peatonal y de bicicletas juega también un papel muy relevante en el marco de las políticas de salud pública, en lo que se refiere a lucha contra la obesidad, la mejora de la calidad del aire y la reducción de muertes por accidente de tráfico, (ROJAS-RUEDA et al, 2011).

En España destacan diversos estudios, como el realizado por Castillo-Manzano y Sánchez-Braza en la ciudad de Sevilla (2013). Una aportación relevante desde la óptica del análisis espacial es la de García-Palomares, Gutiérrez y Latorre (2012), para el caso de Madrid. Los autores utilizan un sistema de información geográfica para optimizar la localización de futuras estaciones del sistema de bicicleta pública, en función de la demanda potencial de usuarios. Cabe citar igualmente algunos manuales de referencia, como el publicado por el IDAE (2007) sobre la implantación de estos sistemas en España.

El presente artículo analiza específicamente el alcance espacial y el impacto en el conjunto de modos de transporte de la implantación de un sistema de bicicleta pública en la ciudad de Palma, BiciPalma, en el marco de un proceso de planificación de movilidad sostenible, iniciado por parte de la administración municipal, hace varios años. La inclusión de la sostenibilidad en el ámbito de la Geografía de los Transportes (MIRALLESGUASCH y CEBOLLADA, 2009) y, específicamente, de la planificación de la movilidad sostenible (BANISTER, 2007) pretende cambiar la cultura de los desplazamientos con la finalidad de reducir los impactos ambientales del transporte a nivel global, incrementar la calidad de vida de los ciudadanos y la accesibilidad a los nuevos modos, dándoles un papel central en la planificación.

Uno de los principales objetivos de los planes de movilidad urbana sostenible, tanto en el marco europeo como en España (HERCE, 2009), estriba en incrementar los desplazamientos en modos no motorizados y en transporte público, para reducir emisiones y facilitar la transición desde el vehículo privado ${ }^{3}$. La estrategia europea de movilidad sostenible a través del cambio modal se perfila en varios documentos, siendo uno de los más destacados, el Libro Blanco del Transporte (2011). Los sistemas de bicicleta pública encajan en los planes de movilidad urbana sostenible al impulsar la movilidad ciclista, sobretodo en ciudades con poca tradición en este tipo de transporte.

El análisis que proponemos pretende pues evaluar el alcance de los sistemas de bicicleta pública en la ciudad de Palma, en base a tres variables principales: la evolución del reparto modal de los desplazamientos en la ciudad; el alcance territorial de la red y el tráfico ciclista generado por el nuevo sistema Finalmente, se abunda en las características de sus usuarios. Para ello se utilizan tres fuentes de datos: dos encuestas de movilidad realizadas por el Consorcio de Transportes de Mallorca y el Ayuntamiento de Palma en 2009 y 2013, respectivamente; los datos de uso de la bicicleta pública obtenidos a través del sistema informático de gestión del Ayuntamiento de Palma; y, por último, los resultados de una encuesta realizada entre los usuarios del sistema en noviembre de 2013.

3 En este marco de movilidad sostenible se inserta precisamente el proyecto europeo CIVITAS-DYN@MO (296057, 2012-2016). 


\section{I.2. Área de estudio}

La ciudad de Palma cuenta con 398.000 habitantes (2013), la mitad de la población de la isla de Mallorca (Islas Baleares). Se erige en centro urbano capital y en cabecera del sistema de transportes, de la red ferroviaria y de carreteras y del Metro. En la ciudad y en su entorno metropolitano se localizan grandes polos generadores de tráfico tales como los hospitales, la universidad, los polígonos de servicios y grandes equipamientos comerciales y de ocio generadores de movilidad. A escala urbana, Palma cuenta con un sistema de transporte público en autobús de 29 líneas, que en 2013 movilizó un total de 38.026.043 pasajeros (datos oficiales de la EMT), estructurado históricamente de forma radial desde el centro de la ciudad hasta la periferia, con algunas pocas líneas diametrales (SEGUÍ y RIPOLL, 1990).

Desde el año 2000 el Departamento de Movilidad del Ayuntamiento de Palma ha desarrollado diversos estudios que, aun no siendo en su integridad Planes de Movilidad Urbana Sostenible tal y como se describen en el marco conceptual y legislativo de referencia (WEFERING et al, 2013) cuentan con actuaciones en materia de movilidad urbana sostenible. En los años 2001 y 2002 se desarrollaron propuestas relacionadas con la construcción de aparcamientos y la peatonalización de algunas calles comerciales en el centro histórico. Posteriormente, en el año 2009, se llevó a cabo un plan de movilidad más ambicioso, en los barrios del ensanche, con diferentes actuaciones relacionadas con la recuperación de los llamados «ejes cívicos» planificados en nuestra ciudad en el urbanismo de los ochenta. Las medidas se centraron en la peatonalización de tramos de calles para crear nuevos espacios públicos y ejes de conexión peatonal y ciclista; además se planificó un incremento importante de la red de carriles bici a través de toda la ciudad (hasta los $66 \mathrm{~km}$., equivalen-

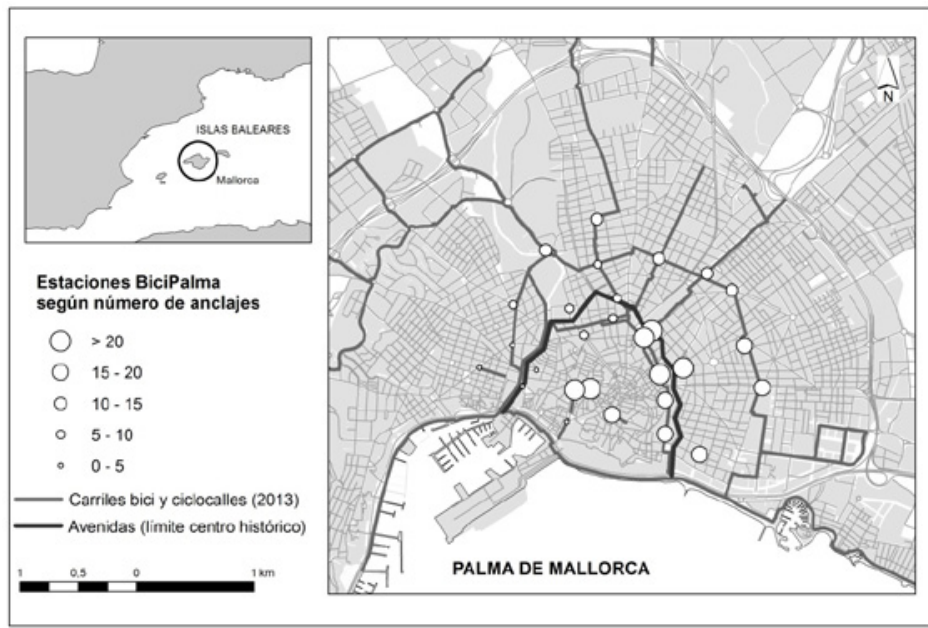

Fuente: Departamento de Movilidad, Ayuntamiento de Palma, proyecto CIVITAS-DYN@MO, elaboración propia. 
Figura 2

ESTACIONES BICIPALMA Y PUNTOS DE RECUENTO DE CICLISTAS

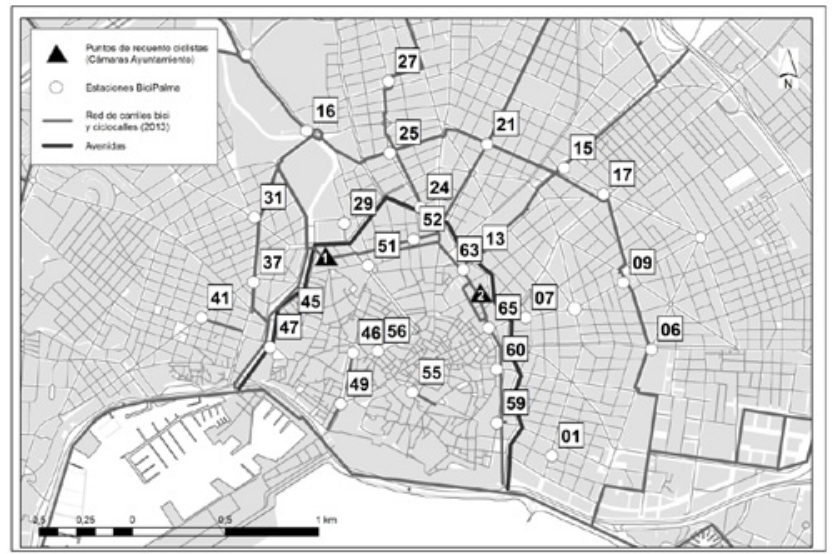

\begin{tabular}{l} 
1. PARC DE SES VELES \\
6. MANACOR - MANUEL AZAÑA \\
7. ARAGÓ-NUREDDUNA \\
8. F. MANUEL DE LOS HERREROS \\
13. PARC DE SES ESTACIONS \\
15. J. VERDAGUER-JAUME \\
BALMES \\
16. PARC DE SA RIERA \\
17. ARAGÓ-JAUME BALMES \\
21. PL. ALEXANDER FLEMING \\
\hline
\end{tabular}

25. BLANQUERNA - P BARTOLOMÉ
27. PL. PARIS
29. INSTITUT BALEAR
31. PL. MADRID
37. AVD ARGENTINA
41. FȦBRICA
45. JAUME III
46. PL. REI JOAN CARLES I
47. PL PORTA SANTA CATALINA
49. PL. DE LA REINA

51. VIA ROMA 52. CECILI METEL

55. PL. SANTA EULALIA

56. PL. DEL MERCAT

59. MATEU ENRIC LLADÓ

60. TRAVESSA D'EN BALLESTER

63. PL. D'ESPANYA

65. PL. ALEXANDRE JAUME

Fuente: Departamento de Movilidad, Ayuntamiento de Palma, proyecto CIVITAS-DYN@MO, elaboración propia.

Tabla 1

CARACTERISTIICAS DE LOS SISTEMAS DE BICICLETA PÚBLICA EN DIVERSAS CIUDADES EUROPEAS

\begin{tabular}{|l|c|c|c|c|c|}
\hline & Estaciones & Bicicletas & $\begin{array}{c}\text { Estaciones / 1.000 } \\
\text { habitantes }\end{array}$ & $\begin{array}{c}\text { Bicicletas / 1.000 } \\
\text { habitantes }\end{array}$ & $\begin{array}{c}\text { Bicicletas / } \\
\text { estación }\end{array}$ \\
\hline Palma & $\mathbf{2 8}$ & $\mathbf{3 0 0}$ & $\mathbf{0 , 1}$ & $\mathbf{0 , 8}$ & $\mathbf{1 0 , 7}$ \\
\hline Barcelona & 420 & 6.000 & 0,3 & 3,7 & 14,3 \\
\hline Zaragoza & 130 & 1.300 & 0,2 & 1,9 & 10,0 \\
\hline Valencia & 275 & 2.750 & 0,3 & 3,5 & 10,0 \\
\hline Sevilla & 250 & 2.500 & 0,4 & 3,6 & 10,0 \\
\hline Lyon & 343 & 4.000 & 0,7 & 8,3 & 11,7 \\
\hline Niza & 171 & 1.200 & 0,5 & 3,5 & 7,0 \\
\hline París & 1.451 & 20.600 & 0,6 & 9,2 & 14,2 \\
\hline Bruselas & 305 & 3.700 & 0,3 & 3,2 & 12,1 \\
\hline Londres & 718 & 9.100 & 0,1 & 1,1 & 12,7 \\
\hline Stuttgart & 75 & 400 & 0,1 & 0,7 & 5,3 \\
\hline Viena & 94 & 1.200 & 0,1 & 0,7 & 12,8 \\
\hline
\end{tabular}

Fuente: elaboración propia a partir de datos del Ayuntamiento de Palma y Metrobike (2014): http://www.metrobike.net/ 
tes a $0,2 \mathrm{~km}$ por cada 1.000 habitantes); la potenciación de la red de autobuses urbanos y el incremento de frecuencias; $y$, finalmente, el desarrollo de un proyecto de tranvía urbano entre el aeropuerto de Son Sant Joan y el centro de la ciudad nunca realizado.

Como complemento a todas estas actuaciones en el mes de marzo del año 2011 se puso en funcionamiento BiciPalma, un sistema de bicicleta pública con 28 estaciones, 485 anclajes y 300 bicicletas (figura 1 y figura 2), de dimensiones ligeramente inferiores a otras ciudades del ámbito estatal o europeo, tanto en lo referido al número de bicicletas por mil habitantes $(0,8)$ como en el número de estaciones $(0,1$ por 1000 habitantes) (tabla 1$)$. Sevilla, Barcelona o Valencia triplican en términos relativos la dotación de Palma.

\section{METOdOLOGÍA}

Las encuestas de movilidad disponibles para los años 2009 (Consorcio de Transportes de Mallorca) y 2013 (Ayuntamiento de Palma) en la ciudad de Palma, proporcionan los datos del reparto modal y el número de desplazamientos realizados en un día laborable tipo. La encuesta del 2009 se realizó en el marco de un estudio global de movilidad para el conjunto de la isla de Mallorca, a partir de una muestra muy elevada y para la que se realizaron más de 5.000 entrevistas telefónicas, sólo en el municipio de Palma, estratificadas en función del género y la edad. Para el año 2013, los encuestados fueron 600; en ambos casos se entrevistó a la población mayor de 15 años a través de cuestionarios específicos en los que se preguntaba por el último desplazamiento realizado.

Para la caracterización espacial del sistema público BiciPalma, sujeto central del trabajo, se han obtenido datos de viajes emitidos y recibidos por cada estación durante una semana completa de los años 2012 y 2013. A través de herramientas SIG se han geolocalizado cada una de las estaciones y, partiendo de los tramos viarios digitalizados del proyecto CartoCiudad ${ }^{4}$, se ha estructurado la red viaria de Palma, sobre la que se ha calculado un área de influencia de $300 \mathrm{~m}$. desde cada estación. El cálculo de la población existente en el ámbito de influencia de cada una de las estaciones se ha obtenido a partir de la información desagregada por manzanas proporcionada por el Ayuntamiento de Palma para el año 2013. Procedimiento análogo al que se ha utilizado para calcular la cobertura de la red de carriles bici.

Para obtener los perfiles y características de los usuarios del sistema se han utilizado los datos proporcionados por los gestores de la bicicleta pública, complementados con una encuesta en línea, abierta entre los días 12 y 26 de noviembre de 2013, enviada por correo electrónico a todos los usuarios de BiciPalma, cuantificados en 2.224 en este periodo, de la que se obtuvieron un total de 716 respuestas válidas (32\% del universo). La encuesta permite obtener un perfil de usuario por género, edad y nivel socioeconómico, a la vez que indagar en los detalles del último desplazamiento realizado y obtener la opinión general sobre el funcionamiento de este modo de transporte.

4 Disponibles en http://www.cartociudad.es 
Finalmente se han utilizado datos de recuentos mensuales de ciclistas realizados por el Ayuntamiento de Palma en dos puntos concretos ubicados en la zona centro, sobre carriles bici ya existentes (figura 2). Se realizan a partir de grabaciones de videocámara entre las 8:00 y las 20:00 horas, en un día laborable de cada mes y recogen el tráfico por hora del número de bicicletas a su paso por cada uno de los controles establecidos.

\section{RESULTADOS}

\section{III.1. Evolución del reparto modal en la ciudad de Palma}

Como característica general, los datos derivados de las encuestas de movilidad del 2009 y 2013 (tabla 2) indican una disminución global del número de desplazamientos realizados en la ciudad de Palma, que pasan de 1.039 .605 a 936.900 (-9,9\%) entre los dos años de referencia y que decrecen en un 25\% si nos remontamos al 2003. La disminución en términos absolutos y relativos de los desplazamientos en vehículo privado (-17\%) es principalmente lo que explica ese descenso. Los movimientos en vehículo privado, del 50,0\% en 2009, pasan a ser del 46\% en 2013, en favor, en el mismo periodo, de un mayor porcentaje de uso del transporte público $(12, \%$ vs $13,0 \%)$ y sobretodo del transporte no motorizado (38\% vs 41, \%). Destaca muy claramente el crecimiento en solitario de los desplazamientos ciclistas que, de los 11.370 movimientos en 2009 aumentan hasta los 18.738 en 2013, con más del 60\% de incremento. Es un claro exponente de los efectos de la expansión del carril bici cuya extensión era de tan sólo 26 kms. en 2008 mientras en 2013 disponía de 66 kms repartidos a lo largo de la ciudad ${ }^{5}$.

$\mathrm{El}$ incremento del transporte en bicicleta en la ciudad viene igualmente corroborado por los datos obtenidos de los recuentos de ciclistas existentes en los dos puntos anteriormente mencionados, ubicados en el centro histórico (figura 2 y figura 3). Estos datos evidencian cómo se alcanzan puntas de tráfico de más de 1.200 ciclistas por día, con diferencias mensuales que indican una menor afluencia en los meses de invierno (febrero, marzo) y verano (agosto).

Tabla 2

EVOLUCIÓN DEL REPARTO MODAL EN LA CIUDAD DE PALMA (\%) (2009-2013)

\begin{tabular}{|l|c|c|}
\hline & $\mathbf{2 0 0 9}$ & $\mathbf{2 0 1 3}$ \\
\hline Peatonal & 36,6 & 38,5 \\
\hline Bicicleta & 1,1 & 2,0 \\
\hline Transporte público & 12,3 & 13,0 \\
\hline Coche & 46,3 & 42,5 \\
\hline Moto & 3,4 & 3,5 \\
\hline Otros & 0,3 & 0,5 \\
\hline Total & $\mathbf{1 0 0 , 0}$ & $\mathbf{1 0 0 , 0}$ \\
\hline
\end{tabular}

Fuente: Consorcio de Transportes de Mallorca y Ayuntamiento de Palma (Diagnóstico del Plan de Movilidad Urbana Sostenible, 2014, Departamento de Movilidad de Palma (CIVITAS-DYN@MO).

5 Diagnóstico del Plan de Movilidad Urbana Sostenible de 2014, Ayuntamiento de Palma. 
Figura 3

EVOLUCIÓN MENSUAL DEL TRÁFICO DIARIO DE CICLISTAS EN DOS PUNTOS DE CONTROL DEL CENTRO HISTÓRICO DE PALMA (2012 Y 2013)

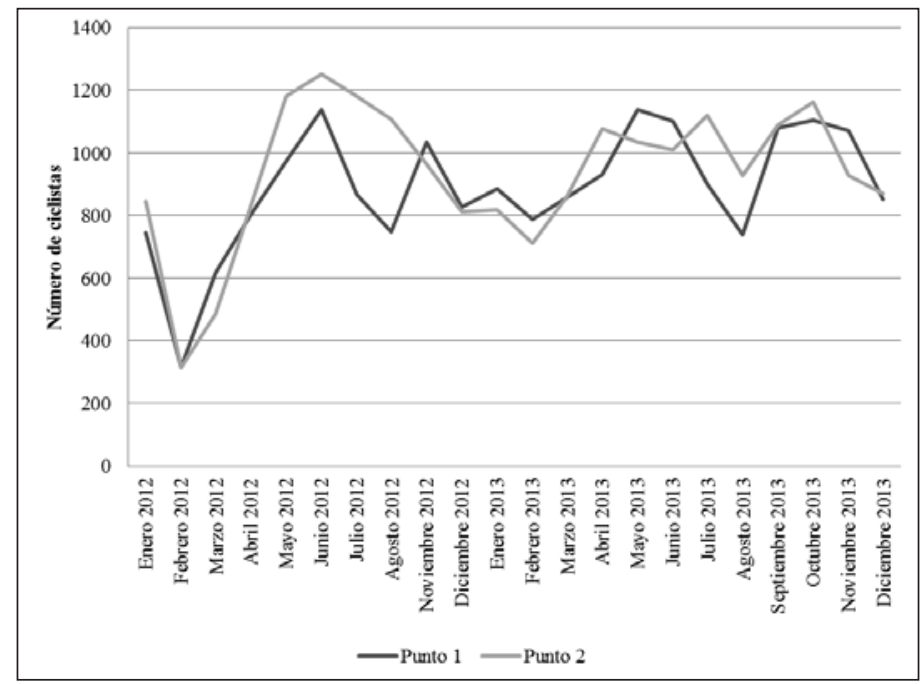

Fuente: Departamento de Movilidad, Ayuntamiento de Palma (CIVITAS-DYN@MO), elaboración propia.

\section{III.2. Cobertura espacial y usos del sistema de bicicleta pública BiciPalma}

El diseño del sistema BiciPalma se fundamentó en la ubicación de estaciones cercanas al centro histórico y a la zona del ensanche más próxima al mismo. La construcción de la red de carriles bici sigue la morfología urbana, radiocéntrica, de Palma, si bien existen algunos itinerarios diametrales de carácter periférico.

La cobertura que ofrece el sistema, en cuanto a población residente situada a menos de 300 m. de una estación BiciPalma, alcanza los 98.896 habitantes (2013), una cuarta parte de la población de Palma. Por el contrario, la cobertura territorial (superficie urbana cubierta por el sistema) es muy reducida y se encuentra por debajo del $10 \%$ de la superficie urbana del municipio (figura 4). Comparativamente, la cobertura ofrecida por la red de carriles bici para la población ubicada a 300 metros de la red, es mucho mayor, pues alcanza a 187.352 personas, casi el $50 \%$ de los residentes en la ciudad.

Los datos analizados durante la semana laborable objeto de estudio, entre los días 6y 10 de Mayo del 2013 (de lunes a viernes), muestran cómo se realizaron un total de 3.058 viajes, lo que supone una media diaria de 611 movimientos para todo el sistema, 21 desplazamientos diarios de media por estación y 0,3 viajes diarios por usuario. La información desagregada por estaciones permite analizar la distribución de los movimientos generados y emitidos. El detalle de los desplazamientos centrífugos y centrípetos de cada estación indica como la media del número de viajes diarios realizados oscila entre los 15,6 y los 105,2 (tabla 3). Dos estaciones se singularizan por contar con más de 80 viajes/día: Plaza París $(105,2)$, ubicada en el extremo norte de la red, y Plaza de España (83), punto neurálgico de la red de transporte público de la 
Figura 4

COBERTURA DEL SISTEMA BICIPALMA

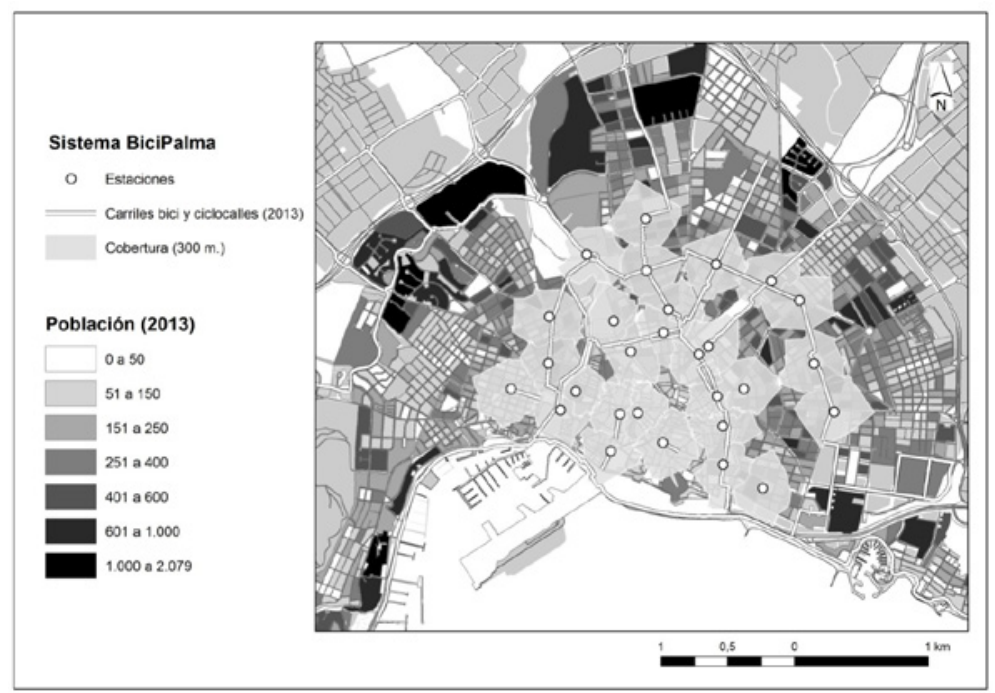

Fuente: Elaboración propia a partir de datos del Departamento de Movilidad, Ayuntamiento de Palma (CIVITASDYN@MO).

Figura 5

MEDIA DE VIAJES/DÍA (EMITIDOS Y RECIBIDOS) POR ESTACIÓN

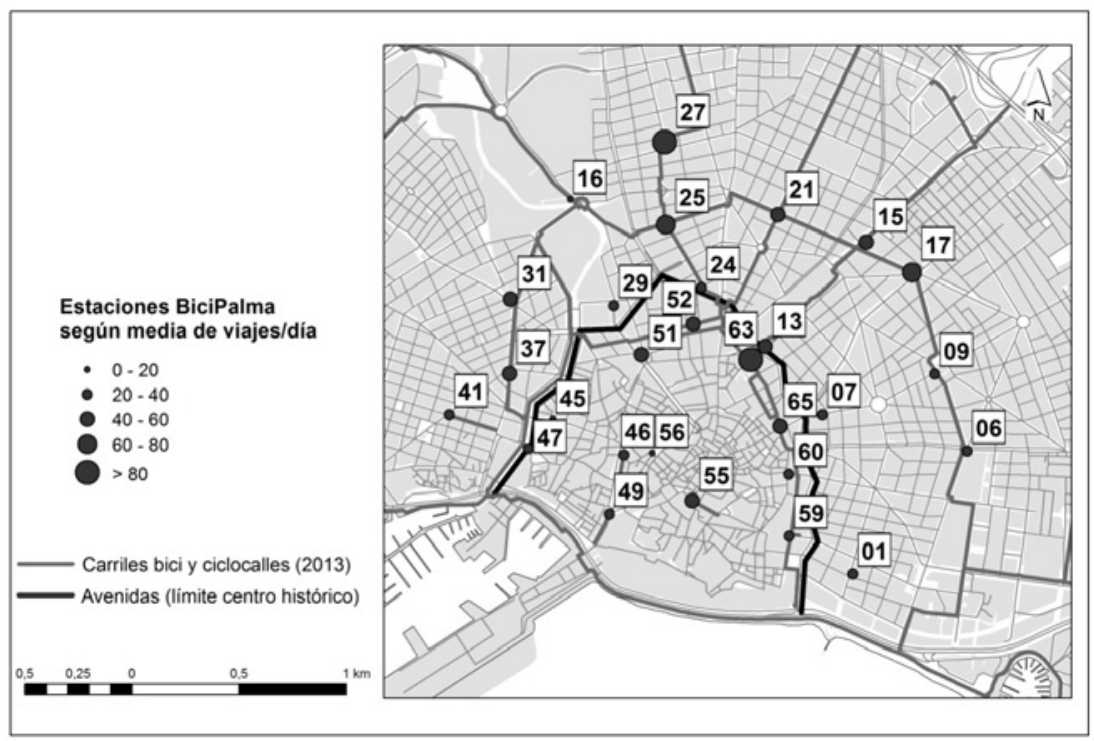

Fuente: Elaboración propia a partir de datos del Departamento de Movilidad, Ayuntamiento de Palma (CIVITASDYN@MO). 


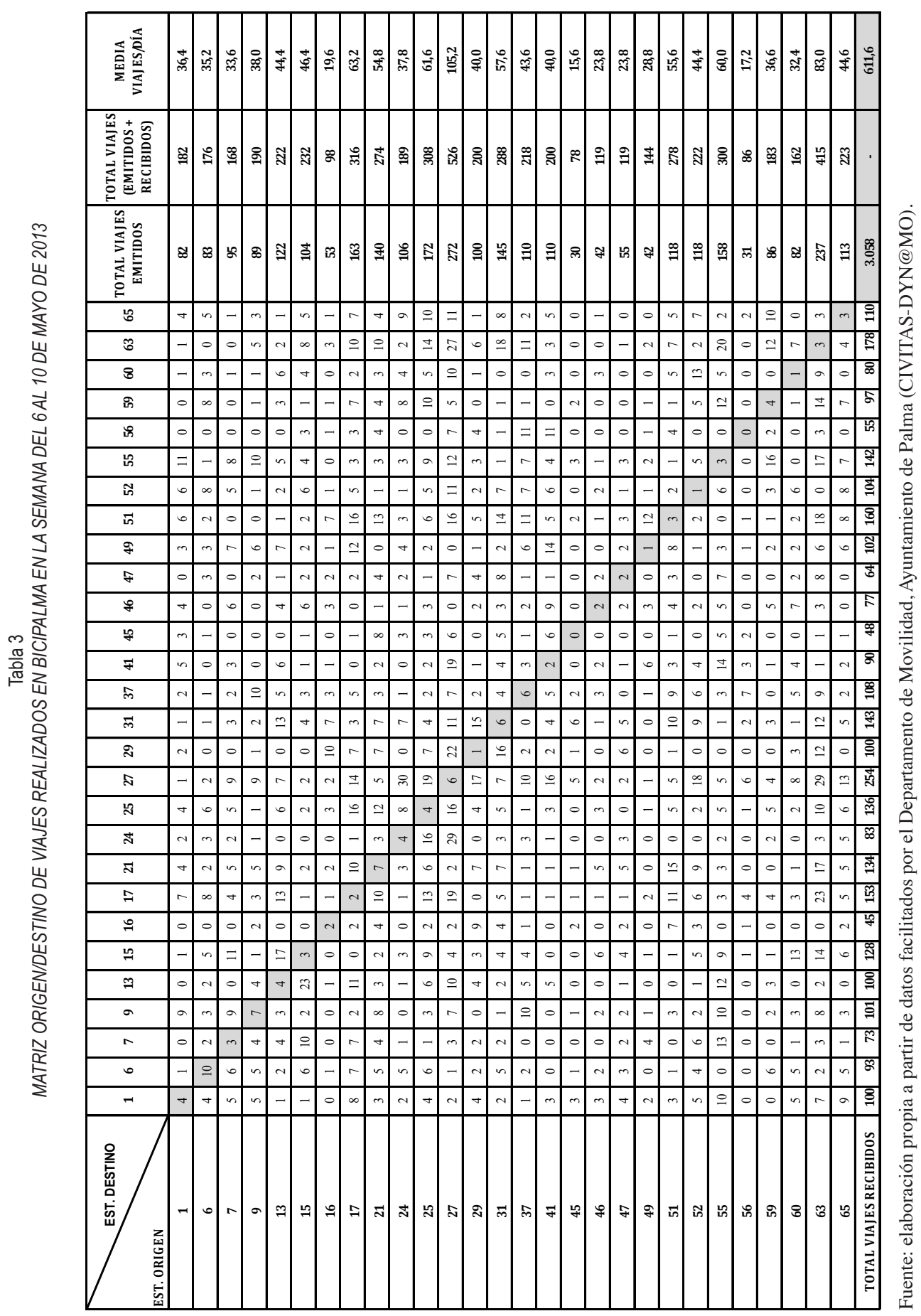


ciudad. Plaza Santa Eulària, con 60 viajes, aparece como el punto más frecuentado en el interior del centro histórico, seguido de la estación de Vía Roma (55), punto de entrada al centrociudad. Las estaciones ubicadas en el cinturón exterior paralelo a avenidas, (Plaza Madrid, Blanquerna-Bartolomé Pou, Alexander Fleming y Aragó-Jaume Balmes) presentan niveles inferiores, de entre 43 y 63 viajes diarios de media (figura 2, figura 5).

Los datos relativos al número de bicicletas prestadas por hora y estación, disponibles para el mismo período de referencia (entre el 6 y el 10 de Mayo del 2013), permiten caracterizar los movimientos entre estaciones según el momento del día y ofrecen una información más exhaustiva de las direcciones de los flujos de usuarios. Un análisis en detalle evidencia, en primer lugar, que las horas punta de tráfico de bicicletas del sistema BiciPalma son las de primera hora de la mañana, entre las 7:00 y las 9:00 horas; y primera hora de la tarde, entre las 13:00 y las 15:00 horas, con dos momentos valle, entre las 10:00 y las 12:00 horas y entre las 16:00 y las 18:00 horas (figura 6).

Un análisis más minucioso de la información de movimientos, desagregados por horas y estaciones, indica cómo los flujos de desplazamientos periferia-centro se dan a primera hora del día (entre las 7:00 y las 9:00 horas), mientras se invierten en dirección contraria, centroperiferia, a partir de las 13:00 horas. La ratio «viajes emitidos/viajes recibidos» permite, a su vez, caracterizar las estaciones según sean emisoras o receptoras de viajes El movimiento es centrípeto a primera hora de la mañana en las estaciones periféricas que son claramente emisoras de movimientos, con ratios generalmente superiores a 2, mientras que las estaciones centrales son claramente receptoras, con ratios por debajo de 0,4. En el período central del día, entre las 13:00 y las 15:00 horas, la situación en términos generales se invierte, con un movimiento centrífugo, suponiendo tasas superiores a 1 para las estaciones del centro e inferiores a 1 para las más periféricas (figura 7).

Figura 6

DISTRIBUCIÓN HORARIA DEL NÚMERO DE VIAJES REALIZADOS

EN BICICLETA PÚBLICA BICIPALMA (6/5/2013A 10/5/2013)

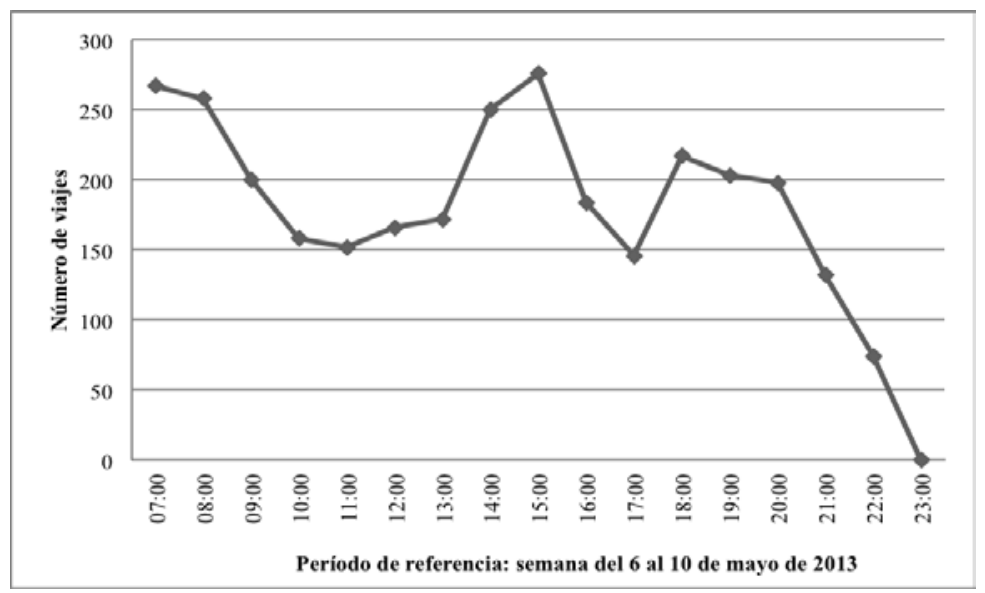

Fuente: Elaboración propia a partir de datos del Departamento de Movilidad, Ayuntamiento de Palma (CIVITASDYN@MO). 


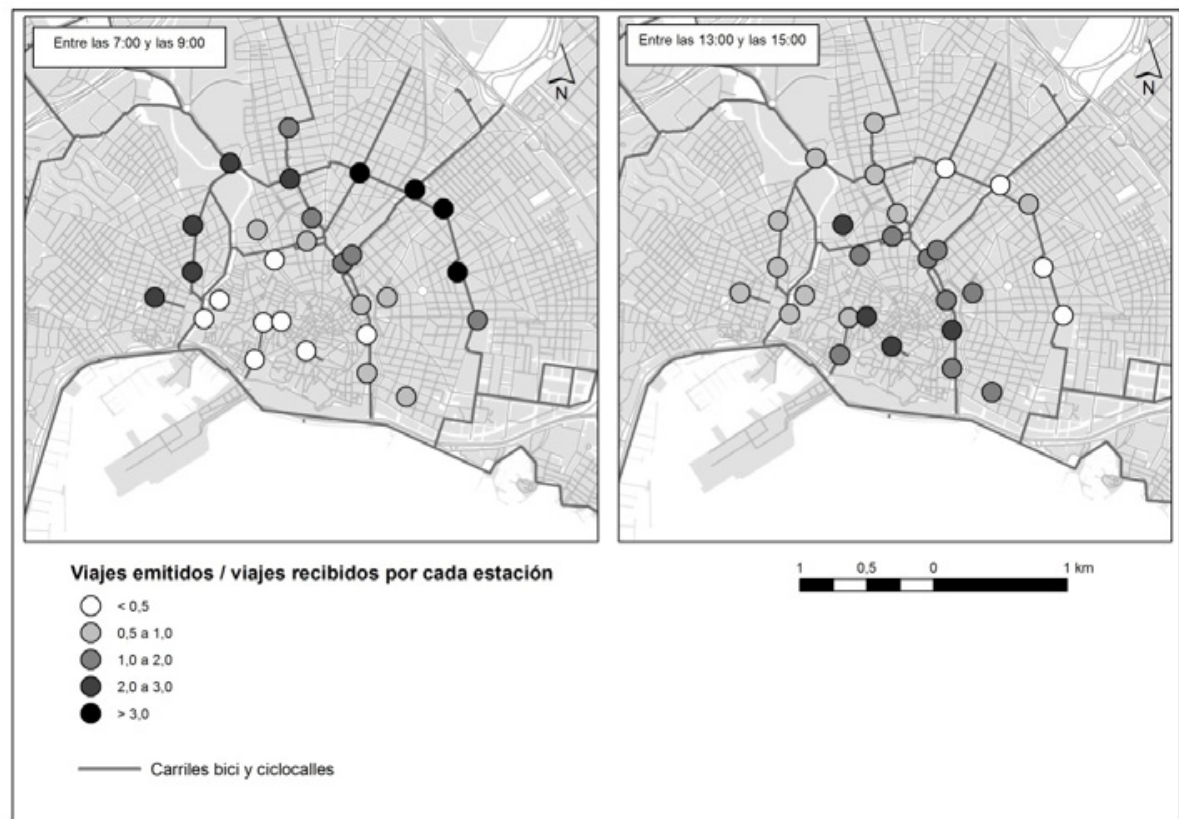

Fuente: Elaboración propia a partir de datos del Departamento de Movilidad, Ayuntamiento de Palma (CIVITASDYN@MO).

\section{III.3. Características de los usuarios}

El perfil de los usuarios de BiciPalma, derivado de la encuesta realizada en Noviembre de 2013 , indica una mayor presencia de hombres que de mujeres (60\% vs 40\%) (figura 8), con tramos de edad, principalmente, entre 35 y 55 años (54\%) y 25-34 años (26\%) y mayoritariamente con un nivel de estudios universitarios (77\%). La mayor parte de usuarios (61\%) son trabajadores por cuenta ajena y tan sólo el $15 \%$ son trabajadores autónomos o empresarios. Únicamente un $8 \%$ están en paro y solamente el 3\% corresponde al segmento de jubilados/ pensionistas.

Si comparamos los resultados por género de los usuarios de BiciPalma, con los generales de la movilidad en Palma, en 2009, observamos cómo son las mujeres las que realizan el mayor número de desplazamientos en modos no motorizados, sin embargo es la marcha a pie la predominante y no la bicicleta. En la misma línea, en la Encuesta de Hábitos de Movilidad desarrollada en la Universidad de las Islas Baleares, los desplazamientos en bicicleta, aunque muy minoritarios por la disposición periférica del campus, representan el 1,5\% de los viajes realizados por los hombres y sólo el 0,3\% por las mujeres (SEGUí y RUIZ, 2014).

En cuanto al uso del sistema BiciPalma, la encuesta indica cómo tan sólo el 20\% de los usuarios utiliza el sistema cada día. El $34 \%$ lo hace dos o tres veces por semana, mientras 
que el $12 \%$ tan sólo una vez semanalmente. Un 34\% de los encuestados utilizan el sistema de manera ocasional (figura 8). Por otra parte, los motivos principales de desplazamiento son: acudir al trabajo (31\%), gestiones personales (30\%) y ocio (22\%). Los movimientos motivados por compras (3\%) y estudios (3\%) son muy poco habituales. En la encuesta se incluyó una pregunta abierta a varias opciones de respuesta sobre el motivo por el que elegían el sistema de bicicleta pública para su desplazamiento. En este sentido, las razones relacionadas con la comodidad («es más cómodo», «más rápido», etc.) son claramente las más frecuentes $(56 \%)$, por encima de los motivos puramente ambientales o de concienciación de una movilidad sostenible (19\%). Únicamente el 5\% de las respuestas señalaba el no disponer de coche como razón primordial para utilizar BiciPalma (figura 8). Estos resultados contrastan con los ofrecidos, para el conjunto de ciclistas, por el Ayuntamiento de Palma, en el documento de diagnóstico del Plan de Movilidad Urbana Sostenible (AYUNTAMIENTO DE PALMA, 2014). Efectivamente, el PMUS aporta datos sobre motivaciones y causas de uso de la bicicleta obtenidos a través de encuestas a ciclistas, en los que la conciencia ambiental («contamina menos») y la comodidad («es muy difícil aparcar») se aducen por parte de un $30 \%$ de los encuestados. Sin embargo, en relación al motivo del viaje, el de «ocio/deporte» aparece en un nivel similar al de la encuesta BiciPalma $(23,3 \%)$, si bien la movilidad obligada (trabajo, estudios) alcanza apenas el $17 \%$ de los encuestados.

Figura 8

CARACTERIZACIÓN DE LOS USUARIOS DE BICIPALMA Y MOTIVOS

DE USO DEL SISTEMA SEGÚN ENCUESTA (NOVIEMBRE 2013)
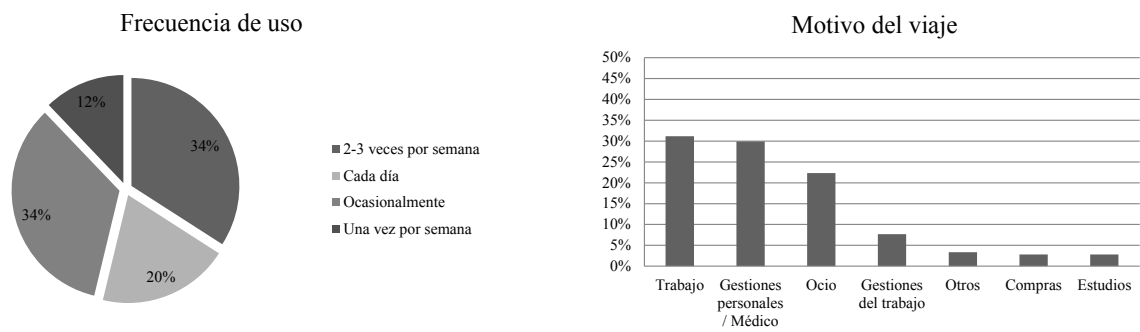

Motivo de uso de BiciPalma

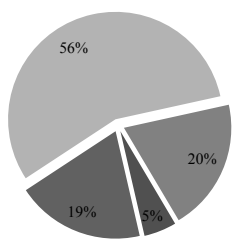

- Motivos ambientales

Motivos comodidad

- Limitaciones

aparcamiento coche

- No dispongo de coche

Encuestados por género y edad

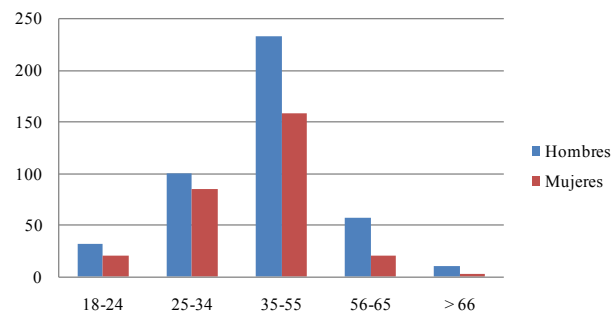

Fuente: CIVITAS-DYN@MO. Grupo de Investigación de Turismo, Movilidad y Territorio (GITMOT). Universitat de les Illes Balears, elaboración propia. 
En relación al lugar de residencia de los usuarios de BiciPalma, el 95\% de las respuestas son de residentes en el propio municipio, y entre el 5\% restante predominan los que viven en municipios cercanos o del área metropolitana de Palma, como Marratxí, Calvià, Consell y Esporles.

Una información complementaria a los movimientos realizados en bicicleta pública es la referida a la cadena modal del viaje llevado a cabo por los encuestados, incluyendo el tramo realizado entre el lugar de origen y la estación BiciPalma. El origen del desplazamiento (el 98\% corresponde a códigos postales del municipio de Palma), para la mitad de los encuestados, se ubica en el mismo código postal de la estación desde la que inician el trayecto. Consecuentemente, el $87 \%$ de los desplazamientos hacia las estaciones BiciPalma se realizan a pie, mientras el resto se realizan en vehículo privado $(7,6 \%)$, mayoritariamente coche, y en transporte público $(5,7 \%)$.

Por último, se insertó en la encuesta un apartado dedicado al grado de satisfacción de los diferentes aspectos del sistema BiciPalma, incluyendo la red de carriles bici de la ciudad. Del análisis de resultados, en una escala del 1 (peor valoración) al 10 (mejor valoración), se desprende que los elementos mejor considerados son los relacionados con la mecánica (estaciones, bicicletas) y con el funcionamiento general del sistema. En este sentido, aspectos como la ubicación de las estaciones $(6,5)$, la disponibilidad de bicicletas en origen $(6,8)$ y el poder contar con anclajes libres en destino $(7,3)$ aparecen bien valorados. También se significan con puntuaciones altas los aspectos vinculados a la información recibida por los usuarios $(6,6)$ o la satisfacción con la respuesta recibida en caso de incidencia $(6,9)$. En cambio, la longitud de la red de carriles bici $(4,6)$, su continuidad $(4,7)$ y la coexistencia con peatones y vehículos motorizados $(4,5)$ son los aspectos peor valorados.

\section{DISCUSIÓN}

El incremento de viajes ciclistas en la ciudad de Palma, tal y como ponen de manifiesto las encuestas de movilidad del Consorcio de Transportes y del propio Ayuntamiento, ha sido notable entre los años 2009 y 2013. Ello es debido, fundamentalmente, a los aumentos constantes a lo largo de estos años de los kilómetros dedicados a carriles bici y a ciclo-calles. Sin embargo, el peso que el sistema de bicicleta pública, BiciPalma, tiene en este incremento es cuantitativamente discutible. Si atendemos a la proporción de los desplazamientos ciclistas realizados en bicicleta pública respecto del total, ésta es más bien baja a tenor de los datos presentados. No obstante, no debe desmerecer el papel de mejora de la imagen de la bicicleta como sistema de transporte cotidiano y habitual que conlleva generalmente la creación de un sistema público y que repercute en la mejora de la eficiencia y la seguridad de los desplazamientos en este modo, y constituye uno de los factores principales en la promoción del transporte en modos no motorizados (FERNÁNDEZ-HEREDIA, MONZÓN y JARA-DÍAZ, 2014).

Los resultados analizados muestran cómo el grado de cobertura y alcance espacial del sistema BiciPalma es bastante limitado. En términos territoriales, supone menos del 10\% del total de la superficie urbana del municipio, lo que permite tan sólo un alcance directo (entendido como la población residente a menos de 300 metros de una estación) del 25\% de la población de la ciudad; comparativamente, contrasta con cerca del $50 \%$ de la población que vive a 300 metros de un carril bici. El uso por parte de residentes ubicados en las zonas 
más periféricas de la ciudad resulta más dificultoso, del mismo modo que por parte de los que habitan fuera del municipio, cuyo acceso aparece condicionado por cuestiones administrativas. A su vez, teniendo en cuenta que el acceso al sistema BiciPalma es para la población mayor de 18 años, el alcance es relativamente bajo.

El impacto en cuanto al tráfico ciclista sobre la ciudad tampoco es demasiado elevado, si tenemos en cuenta que, en un día laborable, se realizan un máximo cercano a los 600 desplazamientos en bicicleta pública. Obviando el número de viajes ciclistas realizados a diario, según la última encuesta de movilidad (más de 18.000,2013) cabe destacar cómo los recuentos de ciclistas en puntos concretos de la red de carriles bici arrojan datos de tráfico cercanos a los 1.200 usuarios diarios, en el mismo período temporal para el que se han recogido datos del sistema BiciPalma. El flujo máximo diario entre dos estaciones BiciPalma es de tan sólo unos 100 viajes para el caso de la estación con más afluencia, movimientos ciertamente reducidos si los comparamos con el tráfico ciclista apreciado en los recuentos reales. La caracterización de usos y desplazamientos permite comprender la dinámica de los flujos entre estaciones. Su distribución horaria encaja con las pautas de tráfico generado por la movilidad obligada: puntas de mayor afluencia a primera hora de la mañana y a mediodía, coincidentes con las horas de entrada y salida al trabajo; y horas valle ubicadas a media mañana y media tarde. Este tipo de flujos coinciden más con horarios laborables de la administración pública u oficinas y despachos diversos pertenecientes al sector terciario que con horas de apertura de comercios, hecho que se corrobora al analizar el perfil y características de los usuarios. Las estaciones periféricas del sistema son más emisoras que receptoras de viajes a primera hora de la mañana, y las de la zona centro reciben más viajes de los que emiten. En las horas centrales del día la situación, por lo general, se invierte, de manera que muchas de las estaciones emisoras se convierten en receptoras. Esta situación concuerda con la distribución horaria del tráfico general de la ciudad.

Del análisis de la encuesta realizada podemos derivar varias conclusiones relevantes. El perfil por género y edad marca una mayor presencia de hombres (60\%) entre 35 y 55 años, lo que difiere de otros casos analizados en los que las franjas de edad más jóvenes son mayoritarias (FISHMAN et al, 2013: 159). Más de la mitad (54\%) de los encuestados tiene entre 35 y 55 años, mientras los que cuentan entre 25 y 35 años, alcanzan apenas el $25 \%$ de los usuarios; la franja de edad de los más jóvenes -que comprende, por ejemplo, los estudiantes universitarios- supone únicamente el $7 \%$ de las respuestas recibidas. En cuanto al nivel socioeconómico y a la situación laboral de los encuestados destacamos la baja presencia de personas en situación de paro (8\%) y el peso relativo de profesionales autónomos y empresarios (15\%). Estas características sociodemográficas se relacionan, a su vez, con que la gran mayoría de los usuarios del sistema (77\%) tiene un nivel de estudios universitarios o equivalente, con empleabilidad superior a la de la población que carece de ellos.

Podemos deducir, pues, que los adultos, mayores de 35 años con un perfil socioeconómico medio, y pertenecientes al género masculino, utilizan más el sistema que las mujeres. Esa diferencia de género, como ya se ha señalado, también se produce en los desplazamientos totales en bici en la ciudad de Palma y en muchas otras ciudades (HEINEN et al, 2010: 69; DILL y VOROS, 2007).

En cuanto a las motivaciones de los desplazamientos, las causas laborales, gestiones varias y estudios son las más aducidas, superiores en conjunto a los movimientos relacionados con el 
ocio. El uso del sistema aparece así mayoritariamente ligado a la movilidad obligada y, hasta cierto punto cotidiana, si consideramos que más del 50\% de los usuarios utilizan el sistema a diario $(20 \%)$ o casi a diario (34\%). En este aspecto, se distancia, tal y como hemos indicado, del conjunto de movimientos en bici en la ciudad de Palma, en el que la movilidad obligada engloba sólo el 17\% de los ciclistas, mientras que el motivo «ocio» alcanza el 23\%. BiciPalma concuerda más con otros casos en Europa en los que se estima que un $46 \%$ de los viajes en bici tienen como destino el lugar de trabajo o estudios (CASTRO, 2011, op cit).

Finalmente, la combinación del uso de la bicicleta con otro modo de transporte público es muy baja: sólo el 5\% de los usuarios acuden a las estaciones a través de este modo, lo que puede venir explicado, en parte, por las dificultades de acceso al sistema por parte de los usuarios no residentes en la ciudad. Los usuarios de BiciPalma viven generalmente cerca de las estaciones, por lo que se desplazan hacia ellas a pie y no precisan de otros modos de transporte para su desplazamiento.

\section{CONCLUSIONES}

El transporte no motorizado se ha convertido en uno de los factores clave a la hora de avanzar hacia un cambio modal que permita aumentar los viajes en modos no motorizados y en transporte público para disminuir la presencia del automóvil en las ciudades y fuera de ellas. La construcción de nuevas infraestructuras para el transporte motorizado como finalidad última de la planificación del transporte, va siendo sustituída por la gestión de la demanda y del sistema de transporte. En la gestión de la demanda de transporte, el aumento de la motorización supera la capacidad de la que dispone el planificador de transporte. Sin embargo, su uso, puede disminuir en función de los diferentes usos del suelo y de las densidades demográficas en las que si inciden los planificadores. La planificación del transporte urbano sostenible y las decisiones políticas para su implementación se materializan en un conjunto de medidas e intervenciones que pueden cambiar y mejorar la habitabilidad de las ciudades. La promoción del uso de la bicicleta se acompaña, entre otras medidas, de: aparcamientos disuasorios, tráfico calmado $(30 \mathrm{~km} / \mathrm{h})$, carriles prioritarios para autobuses y vehículos de alta ocupación, horarios de trabajo alternativos, coche compartido, mejora de las zonas peatonales, mejora del transporte público o la gestión de aparcamientos.

Para mejorar la eficacia y la eficiencia de los sistemas y de las redes de transporte, la gestión de la demanda aparece junto con otras medidas como el pago por el uso de la infraestructura, la utilización de los sistemas inteligentes de transporte, o la gestión de la demanda del tráfico de mercancías, imprescindible para el funcionamiento de las ciudades (RODRIGUE, COMTOIS, SLACK, 2009).

En Palma, la bicicleta pública, puede considerarse un modo de transporte no motorizado minoritario, pero que ha actuado como efecto reclamo en el acusado crecimiento de la movilidad ciclista de Palma en los últimos años, junto con el aumento del número de carriles bici y de aparcamientos específicos para este modo de transporte.

El análisis de la cobertura, funcionamiento y perfil de los usuarios del sistema público BiciPalma pone de manifiesto un alcance todavía limitado. En términos de cobertura, tan sólo llega a una cuarta parte de la población residente y a un $10 \%$ de la superficie urbana del municipio de Palma. En relación al peso de los viajes realizados a través del sistema, la pro- 
porción es todavía baja pues, de media diaria, representa tan sólo un $10 \%$ respecto del total de viajes ciclistas de la ciudad, (100 viajes diarios de media entre dos estaciones BiciPalma respecto de los cerca de 1000 desplazamientos como media de paso en un punto concreto).

El perfil tipo del usuario indica un nivel socioeconómico medio, con un predominio de hombres entre 35 y 55 años y con estudios universitarios. Este tipo de perfil refleja las barreras de acceso al sistema tanto por parte de la población joven (estudiantes) como por parte de las mujeres. La cuestión económica (abono de cuotas) se presume como una de las dificultades principales de acceso al sistema, a tenor del nivel socioeconómico preponderante (sólo un $8 \%$ de los usuarios son parados). Por otra parte, la limitada extensión del sistema y las dificultades de acceso por parte de los no residentes en Palma condicionan también la multimodalidad, es decir su uso combinado con el transporte público, como sistema de acceso al centro de la ciudad desde un aparcamiento periférico. La unimodalidad en la cadena de viaje de la bicicleta pública, a excepción de la marcha a pie, es por lo tanto, mayoritaria.

En el marco de una ciudad en la que la movilidad ciclista se encontraba muy desprestigiada, y en la apuesta global por el impulso de una movilidad más sostenible, BiciPalma ha permitido mejorar la imagen de la bicicleta en la ciudad y ampliar su uso hacia otros perfiles de usuarios que, generalmente, no optaban por este tipo de transporte. A su vez, ha sido un elemento generador de la difusión de la bicicleta como medio de transporte cotidiano y no sólo para su uso en las actividades de ocio.

\section{BIBLIOGRAFÍA CITADA}

AYUNTAMIENTO DE PALMA (2014): Pla de Mobilitat Urbana Sostenible. Departamento de Movilidad. Palma. Disponible en:

http://www.palmademallorca.es/portal/PALMA/Recursos Web/DOCUMENTOS/1/1_81316_2.pdf (consultada en julio 2014).

ALDRED, R. (2013): «Who are Londoners on bikes and what do they want? Negotiating identity and issue definition in a 'pop-up' cycle campaign». Journal of Transport Geography, $\mathrm{n}^{\circ} 30(0)$, pp 194-201.

BANISTER, D. (2008): «The sustainable mobility paradigm». En Transport Policy, 15, pp. 73-80.

CASTILLO-MANZANO, J. y SÁNCHEZ-BRAZA, A. (2013): «Managing a smart bicycle system when demand outstrips supply: the case of the university community in Seville». Transportation, $\mathrm{n}^{\circ}$ 40, pp. 459-477. DOI: 10.1007/s11116-012-9424-7.

CASTRO, A. (2011): The contribution of bike-sharing to sustainable mobility in Europe. Tesis Doctoral. Vienna University of Technology. Disponible en:

http://bicicletapublica.files.wordpress.com/2012/01/dissertation_alberto-castro_1-1.pdf (consultada en abril 2014).

CHEMLA, D.; MEUNIER, F. y WOLFLER CALVO, R. (2013): «Bike sharing systems: Solving the static rebalancing problem». Discrete Optimization, $\mathrm{n}^{\circ} 10$, vol 2. pp 120-146. DOI: http://dx.doi.org/10.1016/j.disopt.2012.11.005

CONSORCI DE TRANSPORTS DE MALLORCA (CTM) (2009). La Mobilitat en dia feiner a Mallorca. Explotació de l'Enquesta de Mobilitat en dia feiner a Palma de Mallorca, 2009. Pdf inédito. 
DILL, J. y VOROS, K. (2007): Factors Affecting Bicycling Demand: Initial Survey Findings from the Portland Region. Transportation Research Board. Washington.

DELL'OLIO, L; IBEAS, A. y MOURA, J.L. (2011): «Implementing bike-sharing systems». Proceedings of the Institution of Civil Engineers. Municipal Engineer, $\mathrm{n}^{\circ} 164$, vol. 2, pp 89-101. DOI: 10.1680/muen.2011.164.2.89

DE MAIO, P. (2009): «Bike-sharing: History, Impacts, Models of Provision, and Future». Journal of Public Transportation, vol. 12, nº 4, pp 41-56.

FERNÁNDEZ-HEREDIA, A.; MONZÓN, A. y JARA-DÍAZ, S. (2014): «Understanding cyclists' perceptions, keys for a successful bicycle promotion». Transportation Research Part A: Policy and Practice, 63, pp. 1-11.

FISHMAN, E.; WASHINGTON, S. y HAWORTH, N. (2012): «Barriers and facilitators to public bicycle scheme use: A qualitative approach». Transportation Research Part F: Traffic Psychology and Behaviour, $\mathrm{n}^{\circ} 15$ vol. 6, pp 686-698. DOI: http://dx.doi. org/10.1016/j.trf.2012.08.002

GARCÍA-PALOMARES, J.C.; GUTIÉRREZ, J. y LATORRE, M. (2012): «Optimizing the location of stations in bike-sharing programs: A GIS approach». Applied Geography, ${ }^{\circ}$ 35, vol. 1-2, pp. 235-246. DOI: http://dx.doi.org/10.1016/j.apgeog.2012.07.002

GOODMAN, A.; GREEN, J. y WOODCOCK, J. (2014): «The role of bicycle sharing systems in normalising the image of cycling: An observational study of London cyclists». Journal of Transport and Health, Vol. 1, $\mathrm{n}^{\circ}$ 1, pp 5-8. DOI: http://dx.doi.org/10.1016/j. jth.2013.07.001

HERCE, M (2009): Sobre la movilidad en la ciudad. Estudios Universitarios de Arquitectura, 18, Barcelona, Editorial Reverté, 321 págs.

HEINEN, E.; VAN WEE, B. y MAAT, K. (2010): «Commuting by bycicle: an overview of the literature». Transport Reviews, vol. 30, $\mathrm{n}^{\circ} 1$, pp. 59-96.

INSTITUTO PARA LA DIVERSIFICACIÓN Y AHORRO DE ENERGÍA-IDAE (2007): Guía metodológica para la implantación de sistemas de bicicletas públicas en España. Ministerio de Industria, Comercio y Turismo. Gobierno de España, Madrid.

MEDDIN, R. y DE MAIO, P. (2014): The Bike-sharing World Map. Recurso electrónico disponible en www.bikesharingworld.com (consultada en abril de 2014).

MIRALLES-GUASCH, C. y CEBOLLADA, A. (2009): «Movilidad cotidiana y sostenibilidad, una interpretación desde la Geografía Humana». Boletín de la Asociación de Geógrafos Españoles, $\mathrm{n}^{\circ}$ 50, pp. 193-246.

MUT I BOSQUE, C. (2010): Valoració de la rendibilitat económica i social del Bicing a Barcelona. Tesina de especialidad. Escola Tècnica Superior d'Enginyers de Camins, Canals i Ports de Barcelona. Universitat Politècnica de Catalunya. Disponible en: http:// upcommons.upc.edu/pfc/bitstream/2099.1/12038/1/Valoraci\%C3\%B3\%20de\%20la\%20 rendibilitat $\% 20$ econ $\% \mathrm{C} 3 \% \mathrm{~B} 2 \mathrm{mica} \% 20 \mathrm{i} \% 20$ social $\% 20 \mathrm{del} \% 20 \mathrm{Bicing} \% 20 \sim 1 . \mathrm{PDF}$ (consultada en abril 2014).

PARKER, S.; MARSDEN, G.; SHAHEEN, S.A. y COHEN, A.P. (2013): «Understanding the diffusion of public bikesharing systems: evidence from Europe and North-America». Journal of Transport Geography, núm. 31 (0), pp 94-103.

RODRIGUE, JP.; COMTOIS,C. y SLACK, B. (2009) The Geography of Transport Systems. New York: Routledge. 
ROJAS-RUEDA, D. ; DE NAZELLE, A. ; TAINIO, M. y NIEUWENHUIJSEN, M. (2011) : «The health risks and benefits of cycling in urban environments compared with car use: health impact assessment study». BMJ (British Medical Journal; 343:d4521.

Disponible en: http://www.bmj.com/content/343/bmj.d4521 (consultada en abril 2014)

SEGUI J.M. y RIPOLL, A. (1990): «Els barris de Palma i la xarxa municipal de transport col.lectiu urbà: un Index d'accessibilitat». Treballs de Geografia, ${ }^{\circ}$ 42. Departament de Ciències de la Terra, Universitat de les Illes Balears.

SEGUÍ, J.M. y RUIZ, M. (2014): Hàbits de mobilitat de la comunitat universitària de la Universitat de les Illes Balears. UIB - Palma. Disponible en: http://gitmot.uib.es/pdf/ mobilitatuib2012.pdf (consultada en julio 2014).

WEFERING, F.; RUPPRECHT, S.; BÜHRMANN, S. y BÖHLER-BAEDEKER, S. (2013): Guidelines. Developing and Implementing a Sustainable Urban Mobility Plan. Directorate-General for Mobility and Transport. Comisión Europea, Bruselas. Disponible en: http://www.mobilityplans.eu/docs/file/guidelines-developing-and-implementing-asump_final_web_jan2014b.pdf (consultada en abril de 2014). 
